\title{
Towards non-equilibrium option pricing theory
}

\author{
Matthias Otto \\ Institut für Theoretische Physik, \\ Universität Göttingen, Bunsenstrasse 9, D-37073 Göttingen, Germany
}

\begin{abstract}
$\underline{\text { Abstract }}$
A recently proposed model (by Ilinski et al.) for the dynamics of intermediate deviations from equilibrium of financial markets ("virtual" arbitrage returns) is incorporated within an equilibrium (arbitrage-free) pricing method for derivatives on securities (e.g. stocks) using an equivalence to option pricing theory with stochastic interest rates. Making the arbitrage return a component of a fictitious short-term interest rate (while the real risk-free rate is assumed to be constant) and thus treating it as another source of uncertainty (besides the security price) in a virtual world, the influence of intermediate arbitrage returns on derivatives in the real world can be recovered by performing an average over the (non-observable) arbitrage return at the time of pricing. Using a famous result by Merton, exact pricing formulas for European call and put options under the influence of virtual arbitrage returns (or intermediate deviations from economic equilibrium) are derived where only the final integration over initial arbitrage returns needs to be performed numerically. This result, which has not been given previously and is at variance with results stated by Ilinski et al., is complemented by a discussion of the hedging strategy associated to a derivative, which replicates the final payoff but turns out to be not self-financing. Numerical examples are given which underline the fact that an additional positive risk premium (with respect to the Black-Scholes values) is found reflecting extra hedging costs due to intermediate deviations from economic equilibrium.
\end{abstract}




\section{Introduction}

The pricing and hedging of derivatives is a major task for financial institutions 14 and has become an increasingly popular topic in statistical physics [7, 8]. Derivatives are sometimes also called contingent claims, as the buyer of the derivatives is entitled to receive a certain payoff up to (or at) some future time $T$, the time of expiry, dependant on the price $S$ of a so-called "underlying" security (say a stock) within a certain time interval between today and $T$ or at time $T$. The simplest case is a "plain-vanilla" European call (put) option which gives the buyer the right to buy (sell) an underlying security (e.g. a stock) at a certain time $T$ in the future for a fixed price $K$ (the strike price).

The classical result of Black and Scholes [1] on option pricing which revolutionized the world of finance and still forms the foundation for most of modern research, is based on the existence of an equilibrium, generally called "absence of arbitrage", i.e. the impossibility of a profit "for nothing".

The use of the no-arbitrage assumption for pricing purposes is nicely elucidated in a standard text book like [14] where simple pricing equations for forward contracts are derived from optimization arguments. If e.g. the forward price $F$ at time $t$ for buying or selling (assuming no bid/offer spreads and transactions costs) a nondividend paying security $S$ at a later time $T$ were less than $S \exp (r(T-t))$, then a riskless profit could be obtained in the following way: at time $t$, one enters into a forward contract to buy the security for $F$ at time $T$, and one short sells the security (i.e. one borrows the security from somebody else and sells it, assuming no fees for simplicity) and puts the proceeds on a deposit at the riskless interest rate $r$ (assuming no credit risk); at time $T$, one receives the security from the forward contract thereby closing out the short position in the security (i.e. handing it over to the lender), while receiving the nominal amount plus interest from the deposit less the forward price paid: $S \exp (r(T-t))-F>0$. Likewise, an arbitrage is possible when $F>S \exp (r(T-t))$. As the information on either situation spreads in the market, the inequalities disappear, and the relation $F=S \exp (r(T-t))$ results. Now the no-arbitrage assumption anticipates the equality to hold right from the beginning, thus implying that arbitrage opportunities disappear infinitely fast. Now many trading activities are motivated exactly by the fact, that this is not the case, but that arbitrage returns exist in the market for a short time $\tau_{\text {arbitrage }}>0$. After this time, the information on arbitrage opportunities has reached enough market participants to make them disappear.

The absence of arbitrage assumption paves the way for one of the fundamental pillars of mathematical finance which is the theorem by Harrison and Pliska [6]. In fact whenever markets are complete (i.e. when any derivative can be hedged by a self-financing strategy, which is a more restrictive statement than absence of arbitrage), then there is a unique equivalent martingale measure for the underlying security and vice versa (see [16] for an introductory discussion on martingale theory). A stochastic process $X_{t}$ is a martingale with respect to measure $\mathrm{Q}$ if and only if $E_{\mathrm{Q}}\left[\left|X_{t}\right|\right]<\infty$

$$
X_{t}=E_{\mathrm{Q}}\left[X_{s} \mid \mathcal{F}_{t}\right], \quad s \geq t
$$

where $\mathcal{F}_{t}$ is the filtration at time $t$, i.e. the information accumulated until time $t$. This rather technical statement is the basis for risk-neutral valuation: Derivatives 
can be priced in a world where all yields are equal to the risk-free interest rate (minus dividend yields etc.).

Deviations from market completeness give rise to the appearance of several possible equivalent martingale measures. The resulting ambiguity for derivatives pricing is then handled by introducing additional constraints on the hedging strategy, e.g. minimizing the expected squared cost for the remaining life time of the option while exactly replicating the final payoff (local risk-minimization) or minimizing the expected squared net loss at the time of maturity of the option (mean-variance hedging) [2], 3, 9] (see also [7, 8] for a physicist's approach). The issue of option pricing in incomplete markets has become a matter of practical interest recently, in particular with the increasing importance of credit derivatives. As opposed to conventional derivatives which cover market risks, there is not a large underlying market of actively traded credit risk instruments in every credit risk category. As opposed to a stock, e.g. a loan is usually not traded.

Considering fully complete and incomplete markets as extreme cases of real markets, one is naturally forced to ask for crossover effects or transitions between the two regimes. A possible answer to this question might be given in terms of a dynamic model which considers market incompleteness in terms of fluctuations around an economic equilibrium characterizing a complete market. An important step in this direction has been given by Ilinski and Stepanenko [4] and Ilinski [5] who assume the existence of intermediate, "virtual" arbitrage returns $x_{t}$, which appear and disappear over a certain time scale which may be identified with $\tau_{\text {arbitrage }}$ mentioned above. In fact, Ilinski et al. intend to treat arbitrage effects as a perturbation to the usual Black-Scholes risk-free rate $r$, which gives the yield on all investments in a risk-neutral world. More specifically, the Black-Scholes risk-free rate is split into a constant part $r^{0}$ and an arbitrage return $x_{t}$ according to $r_{t}=r^{0}+x_{t}$.

In contrast to the line of thought of this article, Ilinski et al. then take the Black-Scholes equation and sum over the dynamics of the arbitrage return $x_{t}$. It is questionable however whether it is correct to take the Black-Scholes equation as the starting point. In fact, this equation is derived on the assumption that the riskfree rate contains no random part. However, Ilinski et al. introduce exactly this forbidden randomness back into the Black-Scholes equation. Moreover, the issue of measure change which is fundamental to option pricing (see e.g. [16]) is not addressed.

Therefore, in this article a different route is proposed. First, the arbitrage return $x_{t}$ is considered as a part of a stochastic interest rate dynamics for the risk-free rate $r_{t}$ in a virtual world (where arbitrage returns are directly observable). Essentially, one is led to a Black-Scholes type equation for a derivative depending on two state variables, the security price $S_{t}$ and the arbitrage return $x_{t}$ which is derived from the risk-free rate in the virtual world according to $r_{t}=r^{0}+x_{t}$. The constant part $r^{0}$ is supposed to be the risk-free rate in the real world which consequently is assumed to be constant. The reason for the latter simplification is the later comparison with the Black-Scholes pricing formulas. As the arbitrage return is an intermediate phenomenon on time scales shorter than the time to expiry, we follow Ilinski et al. by enforcing the boundary condition at the time of expiry of the option that the arbitrage return should disappear. This constraint may be relaxed, however, if one allows for the possibility that any hedging strategy might not replicate (i.e. 
provide for) the final payoff of the option. Nonetheless, we stay with this constraint. However, we do not implement this condition into the payoff function of the option like Ilinski [5], but into the average over arbitrage returns. This procedure allows us to use a famous result by Merton on options in a stochastic interest rate environment [17. After averaging, we obtain a previously unknown exact result for European claims under the influence of virtual arbitrage. (The pricing of American claims which may be exercised prior to their maturity is possible in principle using a "tree" procedure [14, i.e. a scheme based on discrete probabilities and discrete time). These exact pricing formulas differ significantly from the results obtained by Ilinski et al. [4.

The outline of this paper is as follows: In the next section, we present the route from the Black-Scholes model to non-equilibrium market model, taking up the idea of intermediate ("virtual") arbitrage by Ilinski and Stepanenko. The third section will show how the effect of arbitrage returns on option pricing can be considered in terms of a stochastic interest rate environment in a virtual world. In section 4, European call and put options are valued in the presence of virtual arbitrage returns. In section 5 , the issue of a replicating hedging strategy both in the virtual and real world is addressed. Some explicit numerical pricing examples are given in section 6 . In the final section, the results are briefly discussed.

\section{From the Black-Scholes model to the dynamics of arbitrage returns}

Let us briefly review the Black-Scholes analysis in order to motivate the notion of arbitrage returns, following [4]. The model for a one security market is given by

$$
d S_{t}=\mu S_{t} d t+\sigma S_{t} d W_{t}^{1}
$$

where $S_{t}$ is the security price, $\mu$ the drift, and $d W_{t}^{1}$ a Wiener process. It may be motivated from the fact that $\ln \left(S_{i+1} / S_{i}\right)$, where $i+1$ and $i$ denote discrete points in time, performs a random walk [14]. Now the price of a derivative $V_{t}=V\left(S_{t}, t\right)$ whose payoff is contingent on the security price $S_{T}$ at some future time $T$ can be determined by setting up a portfolio $\Pi_{t}$ consisting of the derivative $V_{t}$ and a position $-\Delta$ of the security $S_{t}$ :

$$
\Pi_{t}=V_{t}-\Delta S_{t}
$$

Then if $\Delta=\frac{\partial V}{\partial S}$, where $S=S_{t}$, this portfolio is riskless as uncertainties arising from the Wiener process are eliminated which can be seen by evaluating $d \Pi_{t}$ using Ito's lemma. Therefore, the portfolio is known to grow at the risk-free rate, i.e.

$$
d \Pi_{t}=r \Pi_{t} d t
$$

For constant interest rates, equating expressions for $d \Pi$ gives the Black-Scholes partial differential equation (PDE):

$$
\frac{\partial V}{\partial t}+\frac{\sigma^{2} S^{2}}{2} \frac{\partial^{2} V}{\partial S^{2}}+r S \frac{\partial V}{\partial S}-r V=0
$$


Specifying a certain boundary condition to this equation representing the option payoff at the time of maturity completes the usual Black-Scholes pricing problem. As a reminder, let us note that the drift $\mu$ which was introduced in the market model Eq.(2) is absent from the pricing equation Eq.(5).

The idea of arbitrage returns may be motivated by assuming that in the presence of arbitrage opportunities, the true return of the portfolio $\Pi$ is not equal to the constant risk-free interest rate $r$, but might be less or more than that. Following [5], an arbitrage return $x(t)$ is now introduced by substituting for $r$

$$
r_{t}=r^{0}+x_{t}
$$

where $x_{t}$ is assumed to follow the dynamics of a decay process with random noise:

$$
\frac{\mathrm{d} x_{t}}{\mathrm{~d} t}=-\lambda x_{t}+\eta_{t}
$$

where $\eta(t)$ is characterized by:

$$
\left\langle\eta_{t}\right\rangle=0, \quad\left\langle\eta_{t} \eta_{t^{\prime}}\right\rangle=\Sigma^{2} \delta\left(t-t^{\prime}\right)
$$

As to the nature of $\eta_{t}$ further complications are discussed in [4], but they are not important for our analysis. Basically, a stochastic component $x_{t}$ as been added to the constant risk-free rate $r_{0}$. The question now is: How does the process for the arbitrage return $x(t)$ affect the price of a derivative ?

Substituting Eq.(6) for risk-free rate $r$ in the standard Black-Scholes PDE, Ilinski and Stepanenko simply proceed and derive the following PDE:

$$
\mathcal{L}_{B S} V=x_{t}\left(V-S \frac{\partial V}{\partial S}\right)
$$

where $\mathcal{L}_{B S}$ is the operator from the standard Black-Scholes PDE $\mathcal{L}_{B S} V=0$ for $r=r^{0}$. We will clarify below that the replacement $r \rightarrow r^{0}+x_{t}$ in fact equivalent to introducing an interest rate process $r_{t}=r^{0}+x_{t}$ in a virtual world where tradable instruments dependant on this interest rate exist.

In the next section, a different approach is presented in the framework of standard option pricing theory which allows to study the influence of intermediate deviations from financial equilibrium (as defined by the no-arbitrage assumption) on derivative pricing.

\section{Derivatives in the presence of arbitrage oppor- tunities in terms of option pricing theory with stochastic interest rates}

The stochastic nature of the risk-free rate $r_{t}$ is usually taken into account by stating a stochastic differential equation (SDE) as follows:

$$
d r_{t}=\rho\left(r_{t}, t\right) d t+\Sigma\left(r_{t}, t\right) d W_{t}^{2}
$$


The parameters $\rho$ and $\Sigma$ specify drift and volatility respectively, and may depend on $r_{t}$ and $t$. The drift specifies the deterministic ("trend") component of the interest rate dynamics whereas the volatility describes the stochastic fluctuations. The increment $d W_{t}^{2}$ is a Wiener process. The general PDE for a derivative $V=V(S, r, t)$ dependant on $S=S_{t}$ and $r=r_{t}$ can be found in the literature [14]. Assuming for simplicity no correlations between the Wiener processes $d W^{1}$ from Eq.(2) and $d W^{2}$ and suppressing the functional dependance of $\Sigma$ and $\rho$, the PDE is given by:

$$
\frac{\partial V}{\partial t}+\frac{\sigma^{2} S^{2}}{2} \frac{\partial^{2} V}{\partial S^{2}}+S \frac{\partial V}{\partial S}\left(\mu-\lambda_{1} \sigma\right)-r V+\frac{\Sigma}{2} \frac{\partial^{2} V}{\partial r^{2}}+\frac{\partial V}{\partial r}\left(\rho-\lambda_{2} \Sigma\right)=0
$$

The parameters $\lambda_{i}, i=1,2$ are known as the market prices of risk for the security $S$ and the risk-free rate $r$. They can be obtained by finding the change of measure which makes the respective discounted price process a martingale [16]. For a nondividend paying security governed by Eq.(2), $\lambda_{1}=(\mu-r) / \sigma$. Incidentally, if $r$ is constant, one recovers the Black-Scholes PDE Eq.(5). As $r$ is not a tradable security, a tradable interest rate instrument is needed, e.g. a zero bond with maturity $T$ whose price at time $t$ is $P(t, T)$ and which promises to pay one monetary unit at time $T$. In fact, one is left with a residual freedom of choosing $\lambda_{2}$ [15]. We will return to this issue instantly. Let us now restrict the drift $\rho_{r}$ to a mean-reverting form:

$$
\rho=\rho\left(r_{t}\right)=a-\lambda r_{t}
$$

Moreover, let us suppose that $\Sigma\left(r_{t}, t\right)=\Sigma=$ const. Let us further assume that

$$
r_{t}=r^{0}+x_{t}
$$

Then after transforming from $r$ to $x$ the PDE for the derivative price $V$ reads as:

$\frac{\partial V}{\partial t}+\frac{\sigma^{2} S^{2}}{2} \frac{\partial^{2} V}{\partial S^{2}}+S \frac{\partial V}{\partial S}\left(r^{0}+x\right)-\left(r^{0}+x\right) V+\frac{\Sigma^{2}}{2} \frac{\partial^{2} V}{\partial x^{2}}+\frac{\partial V}{\partial x}\left(a-\lambda r^{0}-\lambda_{2} \Sigma-\lambda x\right)=0$

As pointed out in [15], it is not possible to separate the market price of risk $\lambda_{2}$ from the difference $\tilde{a}=a-\lambda_{2} \Sigma$. Let us now return to the process for the arbitrage return $x_{t}$ assumed in the last section, Eq.(17). Under the martingale measure for the discounted zero bond price, the process for $x_{t}$ obtained from Eq.(10) together with (12) and (13) using standard techniques [16] is given by the SDE:

$$
d x_{t}=\left(\tilde{a}-\lambda r^{0}-\lambda x_{t}\right) d t+\Sigma d \tilde{W}_{t}^{2}
$$

If the equation above is identified with the corresponding expression in Eq.(7), then $\tilde{a}-\lambda r^{0}=0$. Then Eq.(14) has a similar form as Eq.(3) in [5],

$$
\frac{\partial V}{\partial t}+\frac{\sigma^{2} S^{2}}{2} \frac{\partial^{2} V}{\partial S^{2}}+S \frac{\partial V}{\partial S}\left(r^{0}+x\right)-\left(r^{0}+x\right) V+\frac{\Sigma^{2}}{2} \frac{\partial^{2} V}{\partial x^{2}}-\lambda x \frac{\partial V}{\partial x}=0
$$

and the process for the arbitrage return becomes

$$
d x=-\lambda x d t+\Sigma d \tilde{W}_{t}^{2}
$$

yielding the consistency of the no-arbitrage approach in the virtual world with the arbitrage dynamics proposed in Eq.(17). 
Of course, the dynamics of real interest rates are not the same as the dynamics proposed here for a virtual world. The relaxational time scale $1 / \lambda$ originating from the disappearance of virtual arbitrage returns is much shorter than a time scale of mean reversion for real interest rates. As will become clear in the next section, our zero bond price in the virtual world will approach a real (constant interest rate) bond price in the limit of infinitely fast relaxation dynamics for the arbitrage return $x_{t}$. One might object at this point, that we have assumed a hedging strategy in the virtual world which does not exist in the real world ( $x_{t}$ cannot be hedged). Indeed, the hedging strategy in the virtual world expressed in terms of the security $S_{t}$ and a real world cash bond $B_{t}^{0}=\exp \left(-r^{0} t\right)$ leaves us with an extra amount arising from the dynamics of $x_{t}$, and is therefore not self-financing in terms of real world instruments. We will address this issue in more detail in section 5 .

In order to complete the pricing problem in the virtual world, Eq.(16) requires the boundary condition, e.g. for a European claim (which is exercised or not exactly at time $T$ ). It must be chosen as [5]

$$
\left.V(t, S, r)\right|_{t=T}=X \delta(x)
$$

where $X$ is the final payoff (in the real world) depending on $S(T)$ and $r=r^{0}+x$. Then the option price in the real world may be calculated as an average over the initial arbitrage return as follows:

$$
\bar{V}\left(t, S, r^{0}\right)=\int_{-\infty}^{\infty} d x V(t, S, r) \tilde{p}(x)
$$

where $\tilde{p}(x)$ is a probability density function chosen according to the dynamics of $x_{t}$ to be discussed below.

However, we will not proceed to solve the PDE, but remember that according to the Feynman-Kac lemma [10, 11]

$$
V(t, S, r)=E_{\mathrm{Q}}\left[e^{-\int_{t}^{T} d s r_{s}} X \delta\left(x_{T}\right) \mid x_{t}=x, r^{0}, S_{t}\right]
$$

Now it is easy to show (e.g. by using the path integral approach [12]) that

$$
\begin{aligned}
E_{\mathrm{Q}}\left[e^{-\int_{t}^{T} d s r_{s}} X \delta\left(x_{T}\right) \mid x_{t}=x, r^{0}, S_{t}\right] & =E_{\mathrm{Q}}\left[e^{-\int_{t}^{T} d s r_{s}} X \mid x_{t}=x, r^{0}, S_{t} ; x_{T}=0\right] \\
& \times p\left(x_{T}=0 \mid x_{t}=x, r^{0}, S_{t}\right)
\end{aligned}
$$

where $p\left(x_{T}=0 \mid x_{t}=x, r^{0}, S_{t}\right)$ is the conditional probability density function for $x_{T}=0$ given $x_{t}=x, r^{0}, S_{t}$. The last equation allows us to utilize results from the literature on option pricing theory with stochastic interest rates. In fact, we will solve the pricing problem for the actual payoff function $X$ in an interest rate environment where the short rate process in the virtual world $r_{t}$ starts from $r^{0}+x$ at time $t$ and comes back to $r^{0}$ at the time of maturity $T$, or put otherwise where $x_{t}=x$ and $x_{T}=0$.

The average as given in Eq.(19) will then be performed in a different way. From the constraint $\bar{V}\left(t, S, r_{0}\right)=X$, it clear that

$$
\tilde{p}(x)=\frac{p(x)}{p(0)}
$$


where $p(x)$ is the probability density function for the initial value $x$ for the arbitrage return. Using the fact that in our case $p\left(x_{T}=0 \mid x_{t}=x, r_{0}, S_{t}\right)=p\left(x_{T}=0 \mid x_{t}=x\right)$ and

$$
p\left(x_{t}=x \mid x_{T}=0\right)=p\left(x_{T}=0 \mid x_{t}=x\right) \frac{p(x)}{p(0)}
$$

one may rewrite the average in Eq.(19) as follows:

$$
\bar{V}\left(t, S, r^{0}\right)=\int_{-\infty}^{\infty} d x V\left(t, S, r ; x_{T}=0\right) p\left(x_{t}=x \mid x_{T}=0\right)
$$

where

$$
V\left(t, S, r ; x_{T}=0\right)=E_{\mathrm{Q}}\left[e^{-\int_{t}^{T} d s r_{s}} X \mid x_{t}=x, r^{0}, S_{t} ; x_{T}=0\right]
$$

and where $p\left(x_{t}=x \mid x_{T}=0\right)$ is the conditional probability density for the arbitrage return at time $t$ to be equal to $x$ given that its value at $T>t$, the time of expiry, is zero. Its explicit form will be discussed in the next section. In fact, as $t=T$, one obtains $p\left(x_{t}=x \mid x_{T}=0\right)=\delta(x)$ as required.

\section{Valuation of European call and put options in the presence of virtual arbitrage opportunities}

Considering the arbitrage return as a part of the stochastic interest rate $r_{t}$ in our virtual world, we can now draw upon a classical result of Merton [17] in order to derive formulas for European call and put options. In fact, instead of solving Eq.(16) together with Eq.(18) for $X=\max (S-K$; 0 ) (or $X=\max (K-S ; 0)$ ) for call or put options respectively, we consider the security price dynamics Eq.(2) together with the following SDE for the price of a zero bond:

$$
d_{t} P(t, T)=P(t, T)\left(\mu_{P}(t, T) d t+\sigma_{P}(t, T) d W_{t}^{2}\right)
$$

Now assuming that $\sigma_{P}(t, T)$ is a known function of $t$ and $T$, the price of a European call (and put) option $c$ (and $p$ ) at time $t$ which expires at time $T$ with strike price $K$ is given by [17]:

$$
\begin{aligned}
& c=S N\left(d_{1}\right)-P(t, T) K N\left(d_{2}\right) \\
& p=P(t, T) K N\left(-d_{2}\right)-S N\left(-d_{1}\right)
\end{aligned}
$$

where $N(x)$ is the cumulative normal distribution and

$$
\begin{aligned}
d_{1} & =\frac{\ln (S / K)-\ln (P(t, T))+\hat{\sigma}^{2}(T-t) / 2}{\hat{\sigma} \sqrt{T-t}} \\
d_{2} & =d_{1}-\hat{\sigma} \sqrt{T-t} \\
\hat{\sigma}^{2}(T-t) & =\int_{t}^{T} d s\left(\sigma^{2}+\sigma_{P}^{2}(s, T)-2 \rho_{S P} \sigma \sigma_{P}(s, T)\right)
\end{aligned}
$$

The parameter $\sigma$ is the volatility of the security, and $\rho_{S P}$ is the instantaneous correlation between the stock and zero bond prices which for simplicity we assume to be zero as above. Let us now connect to the stochastic interest rate dynamics $r_{t}$. If the 
process for $P(t, T)$ is derived from the process for $r_{t}$ using Ito's lemma, one obtains the following dependance of the bond volatility on the parameters of the process for $r_{t}$ :

$$
\sigma_{P}(t, T)=\Sigma \frac{1}{P(t, T)} \frac{\partial P}{\partial r}
$$

where $\Sigma$ is the short rate volatility. For the specific short rate dynamics chosen in Eq.(10) and (12), the zero bond price can be calculated explicitly as function of $t$, $T$, the current short rate level $r$ and the model parameters. Concerning the latter ones, the drift of the short rate process has to be risk-adjusted by the market price of risk giving $\tilde{a}$ as mentioned above. Let us now calculate the bond price $P(t, T)$. Using the fact that

$$
\begin{aligned}
P(t, T) & =E_{\mathrm{Q}}\left[e^{-\int_{t}^{T} d s r_{s}} \mid r_{0}\right] \\
& =e^{-r^{0}(T-t)} E_{\mathrm{Q}}\left[e^{-\int_{t}^{T} d s x_{s}} \mid x_{t}=x\right]
\end{aligned}
$$

one obtains from the dynamics of $x_{t}$ Eq.(17) (after a tedious calculation given in the appendix using the path integral approach [12]) the following result:

$$
P(t, T)=\exp \left(-\left(r^{0}-\frac{\Sigma^{2}}{2 \lambda^{2}}\right)(T-t)-\frac{1}{\lambda} \tanh \left(\frac{\lambda(T-t)}{2}\right)\left(x+\frac{\Sigma^{2}}{\lambda^{2}}\right)\right)
$$

Performing the differentiation in Eq.(30), one obtains:

$$
\sigma_{P}(t, T)=-\frac{\Sigma}{\lambda} \tanh \left(\frac{\lambda(T-t)}{2}\right)
$$

Now, the restriction $\tilde{a}=\lambda r^{0}$ which makes the drift of the process for the arbitrage return $x_{t}$ be equal to $-\lambda x_{t}$ (under the martingale measure), gives the desired asymptotics of the zero bond price. In fact, as $\lambda \rightarrow \infty$, which can be interpreted as an infinitely fast disappearance of virtual arbitrage returns, it reads as

$$
\lim _{\lambda \rightarrow \infty} P(t, T)=e^{-r^{0}(T-t)}
$$

the zero bond price for a constant risk-free rate $r^{0}$. Therefore the restriction on $\tilde{a}$ mentioned above is reasonable. Let us know turn to the evaluation of the modified security price volatility $\hat{\sigma}$. Evaluating the integral in Eq.(29), one obtains:

$$
\hat{\sigma}^{2}=\sigma^{2}+\frac{\Sigma^{2}}{\lambda^{2}}\left(1-\frac{2}{\lambda(T-t)} \tanh \left(\frac{\lambda(T-t)}{2}\right)\right)
$$

Likewise, in the limit $\lambda \rightarrow \infty$, the contribution to virtual arbitrage returns disappears and one recovers the "bare" security price volatility:

$$
\lim _{\lambda \rightarrow \infty} \hat{\sigma}=\sigma
$$

The asymptotic equations Eq.(34) and (36) assure that in the case of infinitely fast vanishing arbitrage returns the Black-Scholes formulas (for a constant risk-free rate 
$\left.r^{0}\right)$ are recovered from Eq.(27). When the option approaches maturity, there is the following expansion of $\hat{\sigma}^{2}$ :

$$
\hat{\sigma}^{2}=\sigma^{2}+\frac{\Sigma^{2}}{12}(T-t)^{2}+\mathcal{O}\left((T-t)^{3}\right)
$$

Now as the option price in our virtual world is fixed in terms of the parameters of the arbitrage return process, we need to turn to the explicit evaluation of the average carried out in Eq.(24). For an Ornstein-Uhlenbeck process $x_{t}$ given by Eq.(17) it is well known (e.g. [18]), that the transition probability to go from $x^{\prime}$ at time 0 to $x$ at time $t$ is given by:

$$
p\left(x_{t}=x \mid x_{0}=x^{\prime}\right)=\sqrt{\frac{\lambda}{\pi \Sigma^{2}}}\left(1-e^{-2 \lambda t}\right)^{-1 / 2} \exp \left(-\frac{\lambda}{\Sigma^{2}} \frac{\left(x-x^{\prime} e^{-\lambda t}\right)^{2}}{\left(1-e^{-2 \lambda t}\right)}\right)
$$

What is needed however in our case, is $p\left(x_{t}=x \mid x_{T}=0\right)$ for $T \geq t$ which is obtained from Eq.(38) as follows:

$$
p\left(x_{t}=x \mid x_{T}=0\right)=p\left(x_{T}=0 \mid x_{t}=x\right) \frac{p(x)}{p(0)}
$$

where $p(x)$ is the probability density for $x$ which is obtained as a limit probability density form Eq.(38) as $t \rightarrow \infty$ :

$$
p(x)=\sqrt{\frac{\lambda}{\pi \Sigma^{2}}} \exp \left(-\frac{\lambda}{\Sigma^{2}} x^{2}\right)
$$

The final expression for the transition probability thus reads:

$$
p\left(x_{t}=x \mid x_{T}=0\right)=\sqrt{\frac{\lambda}{\pi \Sigma^{2}}}\left(1-e^{-2 \lambda(T-t)}\right)^{-1 / 2} \exp \left(-\frac{\lambda}{\Sigma^{2}} x^{2} \frac{1}{\left(1-e^{-2 \lambda(T-t)}\right)}\right)
$$

It has all the desired features needed. Using the following representation of Dirac's delta function:

$$
\lim _{n \rightarrow \infty} n e^{-\pi n^{2} x^{2}}=\delta(x)
$$

one obtains both for the limit of infinitely rapid disappearance of arbitrage returns

$$
\lim _{\lambda / \Sigma^{2} \rightarrow \infty} p\left(x_{t}=x \mid x_{T}=0\right)=\delta(x)
$$

and for the limit $t \rightarrow T$ of approaching the option's time of maturity

$$
\lim _{t \rightarrow T} p\left(x_{t}=x \mid x_{T}=0\right)=\delta(x)
$$

In both cases, one expects arbitrage returns to disappear. Next, the average over virtual arbitrage returns in Eq.(24) is carried out explicitly for a European call option (for $\bar{V}=\bar{c}$ ) as

$$
\begin{aligned}
\bar{c}\left(t, S, r_{0}\right) & =S \int_{-\infty}^{\infty} d x N\left(d_{1}\right) p(x(t)=x \mid x(T)=0) \\
& -K \int_{-\infty}^{\infty} d x P(t, T) N\left(d_{2}\right) p(x(t)=x \mid x(T)=0)
\end{aligned}
$$


and a European put option (for $\bar{V}=\bar{p}$ ) as

$$
\begin{aligned}
\bar{p}\left(t, S, r_{0}\right) & =K \int_{-\infty}^{\infty} d x P(t, T) N\left(-d_{2}\right) p(x(t)=x \mid x(T)=0) \\
& -S \int_{-\infty}^{\infty} d x N\left(-d_{1}\right) p(x(t)=x \mid x(T)=0)
\end{aligned}
$$

where $P(t, T)$ is given in Eq.(32). The integrations with respect to $x$ cannot be performed analytically. However, the integrands decrease sufficiently fast to zero as $x \rightarrow \pm \infty$, so that a numerical integration can be be easily performed.

It is obvious from intuition that the pricing formulas Eq.s (45) and (46) contain the fundamental time scale $\tau_{\text {arbitrage }}=1 / \lambda$. In fact, one can introduce the following scaled variables:

$$
\begin{aligned}
u & =\lambda(T-t)=\frac{(T-t)}{\tau_{\text {arbitrage }}} \\
r_{\lambda} & =\frac{r}{\lambda} \\
x_{\lambda} & =\frac{x}{\lambda} \\
\hat{\sigma}_{\lambda} & =\frac{\hat{\sigma}}{\sqrt{\lambda}} \\
\sigma_{\lambda} & =\frac{\sigma}{\sqrt{\lambda}} \\
\Sigma_{\lambda} & =\frac{\Sigma}{\lambda^{3 / 2}}
\end{aligned}
$$

Then $\lambda$ can be eliminated from the pricing formulas. The parameters in Eq.(29) can expressed in terms of the scaled variables of Eq.(47):

$$
\begin{aligned}
d_{1} & =\frac{\ln (S / K)-\ln (P(u))+\hat{\sigma}_{\lambda}^{2} u / 2}{\hat{\sigma}_{\lambda} \sqrt{u}} \\
d_{2} & =d_{1}-\hat{\sigma}_{\lambda} \sqrt{u} \\
\hat{\sigma}_{\lambda}^{2} & =\sigma_{\lambda}^{2}+\Sigma_{\lambda}^{2}\left(1-\frac{2}{u} \tanh \left(\frac{u}{2}\right)\right)
\end{aligned}
$$

where $P(u)$ is given by:

$$
P(u)=P(t, T)=\exp \left(-\left(r_{\lambda}^{0}-\frac{1}{2} \Sigma_{\lambda}^{2}\right) u-\tanh \left(\frac{u}{2}\right)\left(x_{\lambda}+\Sigma_{\lambda}^{2}\right)\right)
$$

\section{$5 \quad$ Replicating hedging strategies}

The issue of hedging strategies in the virtual and the real world mentioned above will now be addressed. The fact that there is no instrument in the real world to hedge intermediate arbitrage returns leads us to conjecture that a hedging strategy might not be self-financing.

To be specific, let us denote a cash bond in our virtual world as follows:

$$
B_{t}=\exp \left(\int_{0}^{t} d s r_{s}\right)
$$


It monitors the temporal evolution of the value of an initial cash deposit $B_{0}=1$ which earns the instantaneous interest rate $r_{s}$. Let us further introduce the cash bond in the real world

$$
B_{t}^{0}=\exp \left(r^{0} t\right)
$$

and as a further abbreviation ( which may be termed the "arbitrage bond")

$$
B_{t}^{x}=\exp \left(\int_{0}^{t} d s x_{s}\right)
$$

Evidently, one obtains:

$$
B_{t}=B_{t}^{0} B_{t}^{x}
$$

Taking $B_{t}$ for the moment as a real cash bond, a self-financing strategy $V_{t}$ consists of holding $\varphi_{t}$ in the security $S_{t}$ and $\psi_{t}$ in the cash bond $B_{t}$ such that

$$
V_{t}=\varphi_{t} S_{t}+\psi_{t} B_{t} \Rightarrow d V_{t}=\varphi_{t} d S_{t}+\psi_{t} d B_{t}
$$

i.e. the value change $d V_{t}$ is only due to price changes $d S_{t}$ and $d B_{t}$. For our security price model with stochastic interest rates in the virtual world, one can show that Eq.(54) holds [16]. Moreover $V_{T}=X$, i.e. the value of portfolio $V$ equals the final payoff, i.e. it is replicating. So in terms of our fictitious cash bond $B_{t}$ there is a selffinancing, replicating strategy. In the real world, our strategy will remain replicating by construction (see Eq.s(19) to (24)). However, it will not be self-financing in terms of the real cash bond $B_{t}^{0}$ and the security price $S_{t}$, as can be seen by substituting for $B_{t}$ in Eq.(54):

$$
\begin{aligned}
d V_{t} & =\varphi_{t} d S_{t}+\psi_{t} d\left(B_{t}^{0} B_{t}^{x}\right) \\
& =\varphi_{t} d S_{t}+\psi_{t} B_{t}^{x} d B_{t}^{0}+\psi_{t} B_{t}^{0} d B_{t}^{x} \\
& =\varphi_{t} d S_{t}+\psi_{t} B_{t}^{x} d B_{t}^{0}+\psi_{t} B_{t}^{0} B_{t}^{x} x_{t} d t \\
& =\varphi_{t} d S_{t}+\psi_{t} B_{t}^{x} d B_{t}^{0}+\left(V_{t}-\varphi_{t} S_{t}\right) x_{t} d t
\end{aligned}
$$

The last step was to replace $\psi_{t} B_{t}^{0} B_{t}^{x}=\psi_{t} B_{t}$ by $V_{t}-\varphi_{t} S_{t}$ using Eq.(54). The third term on the r.h.s of the last line accounts for extra costs or gains due to arbitrage opportunities. It is exactly equal to the instantaneous (positive or negative) arbitrage return earned on the delta hedge $V_{t}-\varphi_{t} S_{t}$. In fact, one has $\Delta=\varphi_{t}$, and therefore

$$
\Pi_{t}=V_{t}-\varphi_{t} S_{t}
$$

where $\Pi_{t}$ is the delta hedge portfolio discussed in section 2. The replacement $r \rightarrow$ $r^{0}+x_{t}$ introduced by Ilinski [4] gives rise to the same additional term in the hedging strategy $V_{t}$, if one considers the change $d \Pi_{t}$ as follows:

$$
d \Pi_{t}=\left(r^{0}+x_{t}\right) \Pi_{t} d t=r^{0} \Pi_{t} d t+x_{t} \Pi_{t} d t
$$

The second term on the r.h.s of this equation is the source of additional intermediate profit and loss during the hedging process. Therefore, we conclude that the replacement of Ilinski is completely equivalent to the introduction of a fictitious cash bond $B_{t}$ or likewise an interest rate $r_{t}$ as defined above, which ensures a self-financing hedging strategy in the virtual world.

The additional hedging costs or gains which arise in the real world are covered by an additional premium contained in the option price as obtained in Eq.(24) (with respect to the Black-Scholes price). This premium is positive in most cases as will be clarified below when numerical examples are discussed. 


\section{Some numerical results}

In the following, some results are presented for two market situations, a rather incomplete market (FIG.(1) and (2)) and a fairly complete market (FIG.(3) and (田)). In the first case, the averaged prices $\bar{c}$ (and $\bar{p}$ ), the Black-Scholes prices and the payoff functions at maturity are given, for parameters $\lambda=10, T-t=0.8, \Sigma=2$, $\sigma=0.2, K=100, r^{0}=0.08$. The unit of time is 1 year, so $\lambda=10$ corresponds to the rather long relaxation time $\tau_{\text {arbitrage }}$ of about 25 trading days, supposing a year of 250 trading days. $\Sigma=2$ is inferred from a daily maximum variation of $x_{t}$ of about $20 \%$ in absolute value (at 95\% confidence level) according to the discretized Langevin equation:

$$
\Delta x=x_{t+1}-x_{t}=-\lambda x_{t} \Delta t+X \Sigma \sqrt{\Delta t}
$$

The random variable $X$ is standard normally distributed. Taking $\Delta=1 / 250, X=$ 1.65 representing the two-sided $95 \%$ confidence interval, $x_{t}=0$ (as an initial value), one concludes

$$
\Sigma=9.58 \Delta x
$$

For various times to maturity $T-t$, FIG.(3) and (4) presents differences of $\bar{c}($ and $\bar{p})$ and the Black-Scholes prices for the choice of parameters $\lambda=100, \Sigma=0.4, \sigma=0.2$, $K=100, r^{0}=0.08$. $\lambda=100$ corresponds to a relaxation time of 2 to 3 trading days, whereas $\Sigma=0.4$ is inferred from a daily variation of $x_{t}$ of about $4 \%$ in absolute value.

Let us now comment on the results. Focusing first on the qualitative behavior, over a reasonable range of the moneyness parameter $m=S / K$, the price of a European call or put option ( $\bar{c}$ or $\bar{p}$ respectively) under the influence of virtual arbitrage is higher than the Black-Scholes value (see FIG.(目) and (2)). The difference is more pronounced at the point of maximum curvature which is around $m \simeq 1$ or below, whereas it decreases whenever $m<1$ or $m>1$ (see FIG.(3) and (41)). For $m \gg 1$, the call option price is less than the Black-Scholes value. As the time to expiry increases the positive difference (except for $m \gg 1$ ) increases, and the maximum difference is shifted to lower values of $m$.

Leaving aside for the moment the negative difference appearing for call option at $m \gg 1$, it appears reasonable that the existence of virtual arbitrage returns causes the option price to be above the Black-Scholes value, as deviations from equilibrium in general lead to an increase in hedging costs, i.e. the costs for readjusting a replication portfolio which is supposed to provide for the final payoff of the option. This effect needs to be accounted for in the option premium. The fact that the absolute difference to the Black-Scholes result is the largest at the point of maximum curvature of the pricing function is understandable from the $\Gamma$ ("gamma") risk point of view. $\Gamma$ denotes the second derivative of the option price with respect the asset price $S$ and gives a measure for the non-linear dependance of the option on the underlying asset. This non-linear risk inherent to options can be only be hedged by buying or selling other options. Any deviations from financial equilibrium due to arbitrage opportunities will affect both the option at hand and the options chosen for hedging. Moreover, the additional term arising in Eq.(55) leading to intermediate $\mathrm{P} \& \mathrm{~L}$ during the hedging process is proportional to the delta hedge $V_{t}-\varphi_{t} S_{t}$ which 
is most relevant at the point of maximum $\Gamma$ where the delta hedge is insufficient. Therefore, these numerical results are completely consistent with our mathematical discussion of the hedging strategy.

The influence of intermediate arbitrage returns grows as the time to expiry of the option increases on the scale of $\tau_{\text {arbitrage }}$ (see FIG.(3) and (41)) (all other parameters being constant). Several deviations from equilibrium during the life time of an option seem to accumulate leading to a higher additional risk premium on the option price.

Returning to the issue of the negative difference for call options that are far in the money $m \gg 1$ in FIG.(1) and (3), a possible explanation is an "overheated" market, where deviations from equilibrium tend to relax from the current asset price to a lower equilibrium price. This information is accounted for by pricing the option at a discount with respect to the Black-Scholes value at the current asset price: the market is expected to decrease to a lower price level.

Considering the quantitative differences between $\bar{c}$ (and $\bar{p}$ ) and the Black-Scholes prices for calls and puts, they are obviously more pronounced in an imcomplete market (FIG.(11) and (2)), than in a rather complete market where arbitrage returns are small and relax fast ((3) and (4)). The numerical analysis given here may refined in various ways (according to the parameter dependances of the options prices) which is the subject of future work.

As opposed to our results, e.g. the first order correction to the Black-Scholes prices for calls given in [4] increases monotonously with moneyness $m$ which is at variance with our result.

\section{Conclusion}

Using the (Ornstein-Uhlenbeck type) relaxational dynamics for "virtual" arbitrage returns introduced in [4], we have derived closed formulas for simple ("plain vanilla") European calls and puts in the presence of arbitrage opportunities appearing and disappearing on an intermediate time scale $\tau_{\text {arbitrage }}=1 / \lambda$. This result which has not been derived previously is obtained using arbitrage-free option pricing theory, by making the arbitrage return process part of an interest rate process in a virtual world. The influence on option prices in the real world (in the presence of rapidly appearing and disappearing arbitrage opportunities) is taken into account by summing over the initial arbitrage return, and imposing the constraint that arbitrage is absent at the time of maturity of the option.

Comparing our work to [4, 5], first, we consider the analysis given above as conceptually more clear as to where arbitrage-free pricing fails and where it does not. Therefore, in the present work a different route has been proposed by introducing a second source of randomness in the derivative pricing problem (apart from the security $S$ ) right from the beginning. As a consequence, a two variable version of Ito's lemma must be used, giving a PDE equation for the derivative price in a virtual world which is finally summed over $x(t)$ to yield the real world price. Second, instead of making the constraint that arbitrage return should vanish at maturity a part of the payoff function in the virtual world as in [4], we enforce it when the average over virtual arbitrage return is taken. This procedure allows us to profit from Merton's classical result on option pricing in a stochastic interest rate environment [17 and 
to arrive at closed-form (up to a numerical integration which is easy to perform) pricing formulas for simple European call and put options.

Furthermore it has been shown that any hedging strategy will not be selffinancing in the real world where the arbitrage return is not directly observable. However, on the average any intermediate costs arising during the hedging process are covered by an additional premium contained in the option price.

The present work may be extended in various directions. The relaxational dynamics of the arbitrage return may be considered to be more complicated as proposed here where it follows a simple Ornstein-Uhlenbeck process. However, additional model parameters introduce more sources of model error from the practitioner's point of view as each parameter has to calibrated to the market. Furthermore, the constraint $x_{T}=0$, i.e. that arbitage returns should disappear at the time of maturity of the option, may be relaxed to allow for a hedging mismatch at maturity. This amounts to give up the constraint that the hedging strategy is replicating. The extension of this work to the case of correlations between the asset price $S_{t}$ and the arbitrage return $x_{t}$ is under way.

Acknowledgements: Discussions with M. Wilkens and participants of the "Internes Seminar Wertpapiermanagement" at the Institut für Betriebliche Geldwirtschaft (IfBG), Universität Göttingen are gratefully acknowledged. The author thanks A.K. Hartmann for a critical reading of the manuscript, and acknowledges financial support by the DFG under grant Zi209/6-1.

\section{Appendix}

Following [12] we propose to evaluate the expectation value

$$
I=E_{\mathrm{Q}}\left[e^{-\int_{t}^{T} d s x_{s}} \mid x_{t}=x\right]
$$

The expectation value can be stated in terms of a quotient of path integrals as follows:

$$
I=\frac{\int_{x(t)=x}^{x(T)=0} \mathcal{D} x(s) \exp \left(-\frac{1}{2 \Sigma^{2}} \int_{t}^{T} d s\left(\frac{\mathrm{d} x(s)}{\mathrm{d} s}+\lambda x(s)\right)^{2}-\int_{t}^{T} d s x(s)\right)}{\int_{x(t)=x}^{x(T)=0} \mathcal{D} x(s) \exp \left(-\frac{1}{2 \Sigma^{2}} \int_{t}^{T} d s\left(\frac{\mathrm{d} x(s)}{\mathrm{d} s}+\lambda x(s)\right)^{2}\right)}=\frac{X}{Y}
$$

Now the numerator and the denominator can be mapped to the propagator of the harmonic oscillator in the presence of an external field, and can thus be evaluated [19]. The expression for the numerator reads as

$$
\begin{aligned}
X & =\sqrt{\frac{\lambda}{2 \pi \Sigma^{2} \sinh (a(T-t)}} \exp \left(\frac{\Sigma^{2}}{2 \lambda^{3}}\left(e^{-\lambda(T-t)}-1+\lambda(T-t)\right)\right. \\
& -\frac{\lambda}{2 \Sigma^{2} \sinh (\lambda(T-t))}\left(x^{2} \cosh (\lambda(T-t))\right. \\
& \left.+2\left(e^{\lambda(T-t)}-1\right)\left(C x+C^{2}\right)\right) \\
& \left.+\frac{\lambda}{2 \Sigma^{2}} x^{2}\right)
\end{aligned}
$$


where

$$
C=\frac{\Sigma^{2}}{2 \lambda^{2}}\left(e^{-\lambda(T-t)}-1\right)
$$

Likewise one obtains an expression for the denominator:

$$
Y=\sqrt{\frac{\lambda}{2 \pi \Sigma^{2} \sinh (\lambda(T-t)}} \exp \left(\frac{\lambda}{2 \Sigma^{2}} x^{2}-\frac{\lambda}{2 \Sigma^{2} \sinh (\lambda(T-t))} x^{2} \cosh (\lambda(T-t))\right)
$$

Calculating $X / Y$ gives the result

$$
I=\exp \left(\frac{\Sigma^{2}}{2 \lambda^{2}}(T-t)-\frac{1}{\lambda} \tanh \left(\frac{\lambda(T-t)}{2}\right)\left(x+\frac{\Sigma^{2}}{\lambda^{2}}\right)\right)
$$

which leads to Eq.(32).

\section{References}

[1] F. Black, M. Scholes. J. of Political Economy 81, 637-654, 1973.

[2] H. Föllmer, M. Schweizer. "Hedging of contingent claimes under imcomplete information". In: M. Davis and R. Elliott (eds.), Applied Stochastic Analysis, Stochastic Monographs 5, 389-414. Gordon \& Breach, London/New York.

[3] D. Heath, E. Platen, and M. Schweizer. "Comparison of some key approaches to hedging in incomplete markets". Working paper FMRR98-003, University of Technology Sydney, 1998.

[4] K. Ilinski, A. Stepanenko. "Derivative pricing with virtual arbitrage". Preprint, cond-mat/9902046.

[5] K. Ilinski. "How to account for virtual arbitrage in the standard derivative pricing". Preprint, cond-mat/9902047.

[6] J.M. Harrison, S.R. Pliska. Stochastic Processes and their Applications 11, $215,1981$.

[7] J.P. Bouchaud, D. Sornette. J. Phys. I (France) 4, 863, 1994.

[8] J.P. Bouchaud. "Elements of a Theory of Financial Risks". In: Order, Chance and Risk. Les Houches school (March 1998), Springer/EDP Sciences, to be published. cond-mat/9806101.

[9] S. Fedotov, S. Mikhailov. "Option pricing model for incomplete market". Preprint, cond-mat/9807397.

[10] R. P. Feynman. Rev. Mod. Phys. 20, 367, 1948.

[11] M. Kac. Trans. Amer. Math. Soc. 65, 1, 1949. 
[12] M. Otto. "Using path integrals to price interest rate derivatives". Preprint, cond-mat/9812318.

[13] O.A. Vasicek. "An Equilibrium Characterization of the Term Structure". J. of Financial Economics 5, 177, 1977.

[14] J. Hull. Options, Futures and Other Derivatives. Prentice-Hall International, 1997.

[15] R. Rebonato. Interest-Rate Option Models. John Wiley \& Sons, Chichester, 1996.

[16] M. W. Baxter, A.J.O. Rennie: Financial Calculus. Cambridge University Press, Cambridge, 1996.

[17] R. C. Merton. "Theory of Rational Option Pricing". Bell J. Econ. Management Sci. 4, 141-183, 1973.

[18] W. Feller. An Introduction to Probability Theory and Its Applications. 3rd edition. John Wiley \& Sons, New York, 1968.

[19] R.P. Feynman. Statistical Mechanics. Addison-Wesley, Reading, 1972. 
FIG. 1: The call option price as a function of moneyness $m=S / K$ (dashed curved line: with virtual arbitrage; solid line: Black-Scholes formula). The dashed straight line is the payoff function at maturity. Parameters: $\lambda=10, T-t=0.8, \Sigma=2$, $\sigma=0.2, K=100, r^{0}=0.08$.

FIG. 2: The put option price as a function of moneyness $m=S / K$ (dashed curved line: with virtual arbitrage; solid line: Black-Scholes formula). The dashed straight line is the payoff function at maturity. Parameters: see FIG. 1.

FIG. 3: Difference of the call price to Black-Scholes value (in absolute value) for various values $T-t$. Other parameters: $\lambda=100, \Sigma=0.4, \sigma=0.2, K=100, r^{0}=0.08$.

FIG. 1: Difference of the put price to Black-Scholes value (in absolute value) for various values $T-t$. Other parameters: see FIG. 3 . 


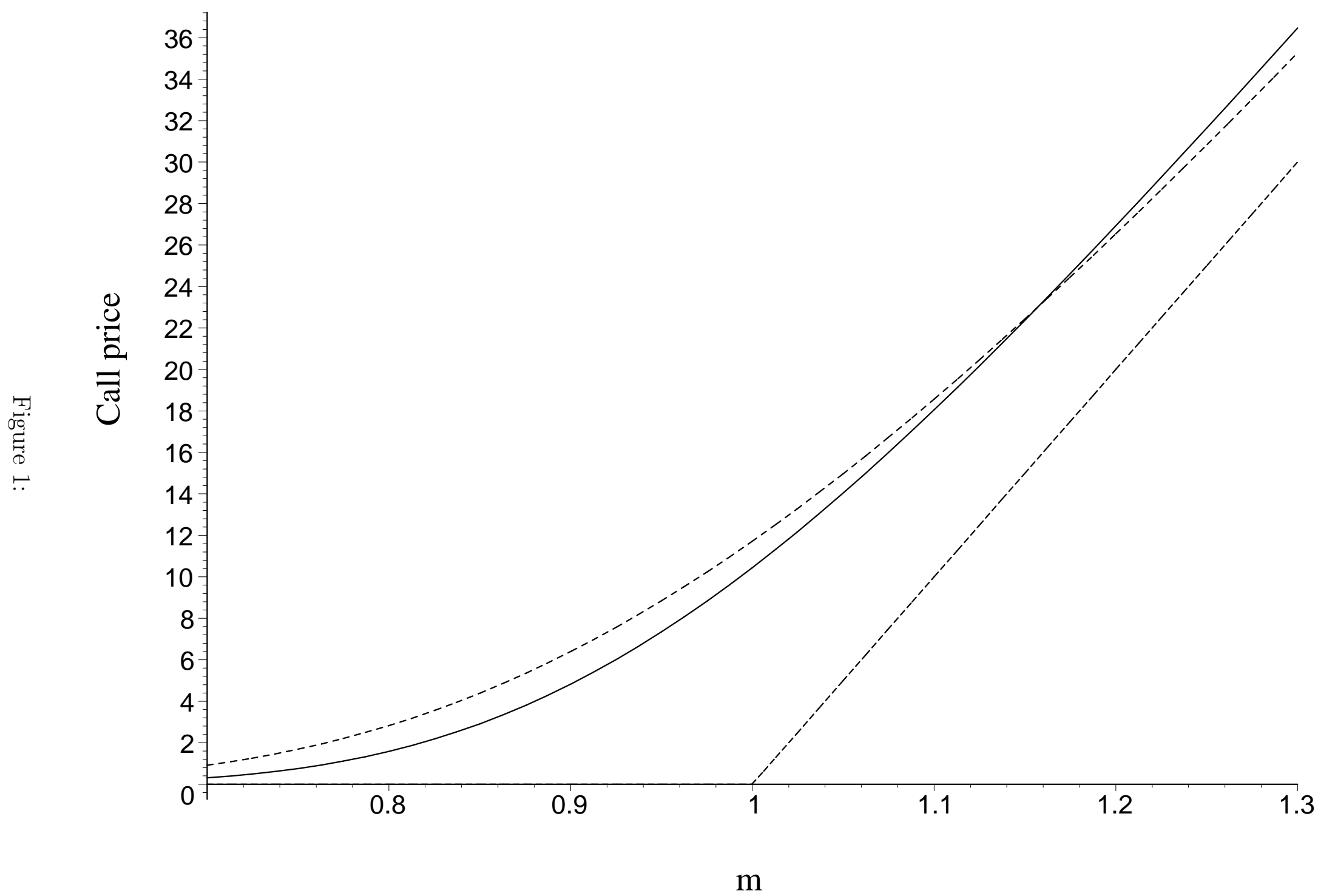




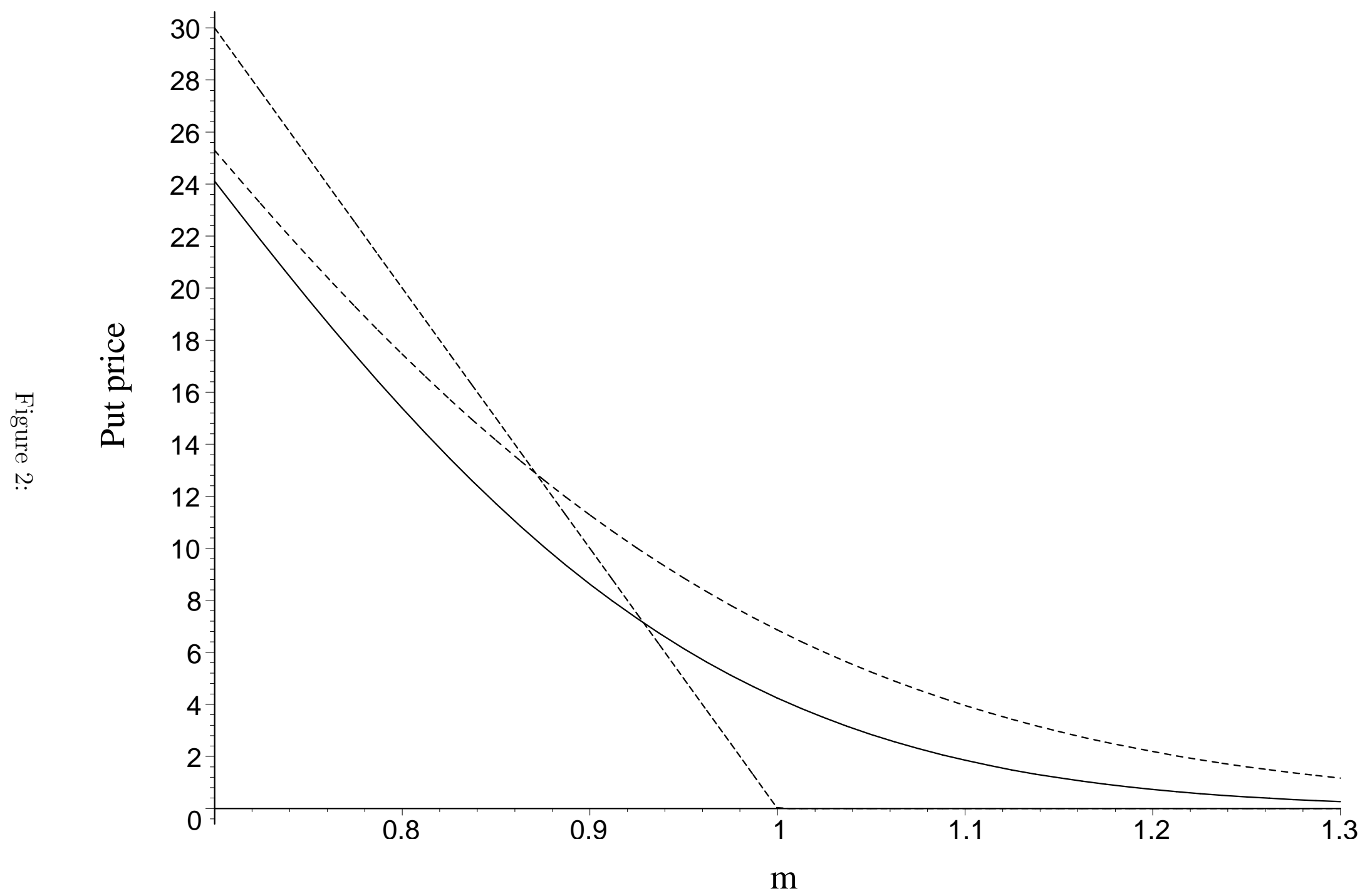




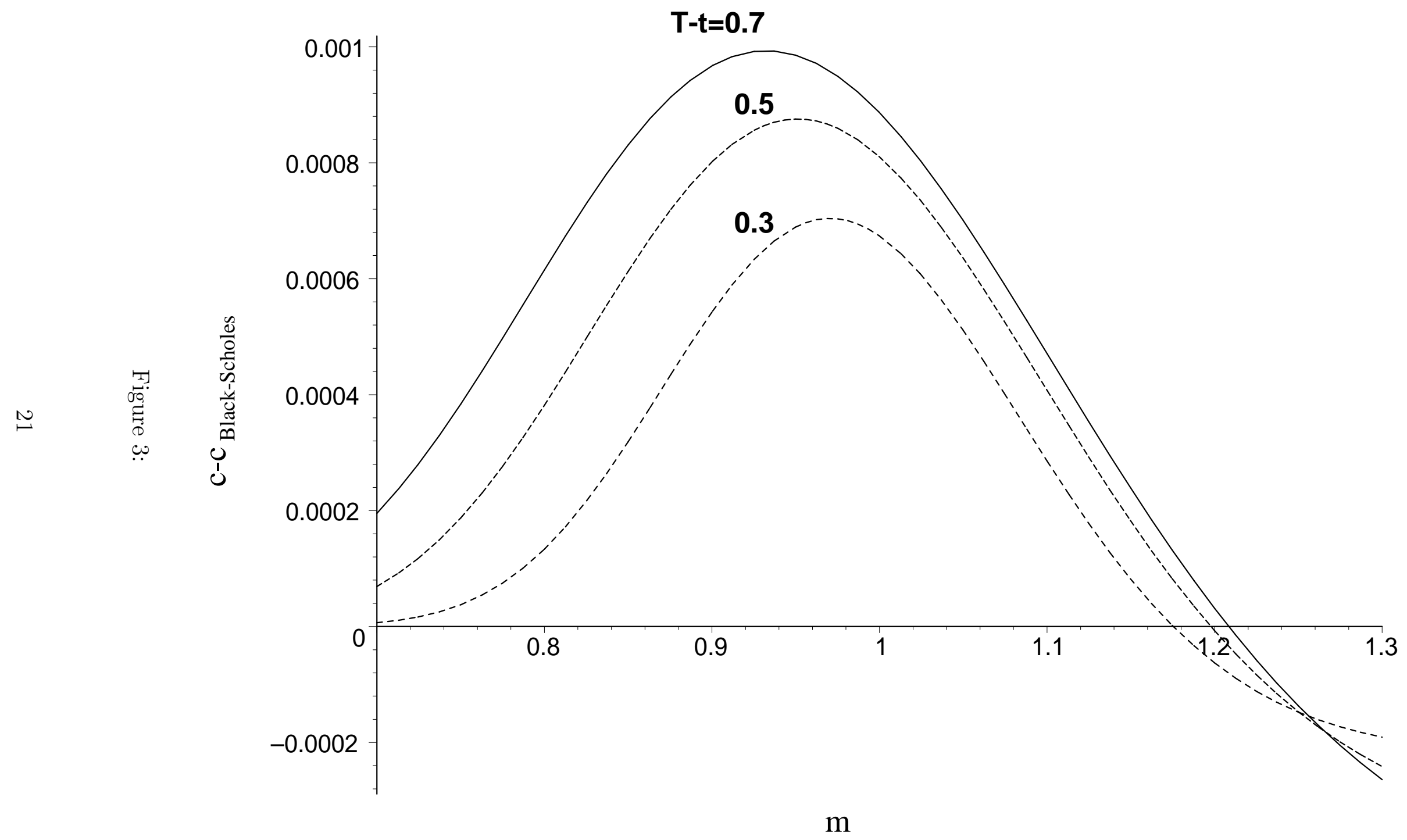




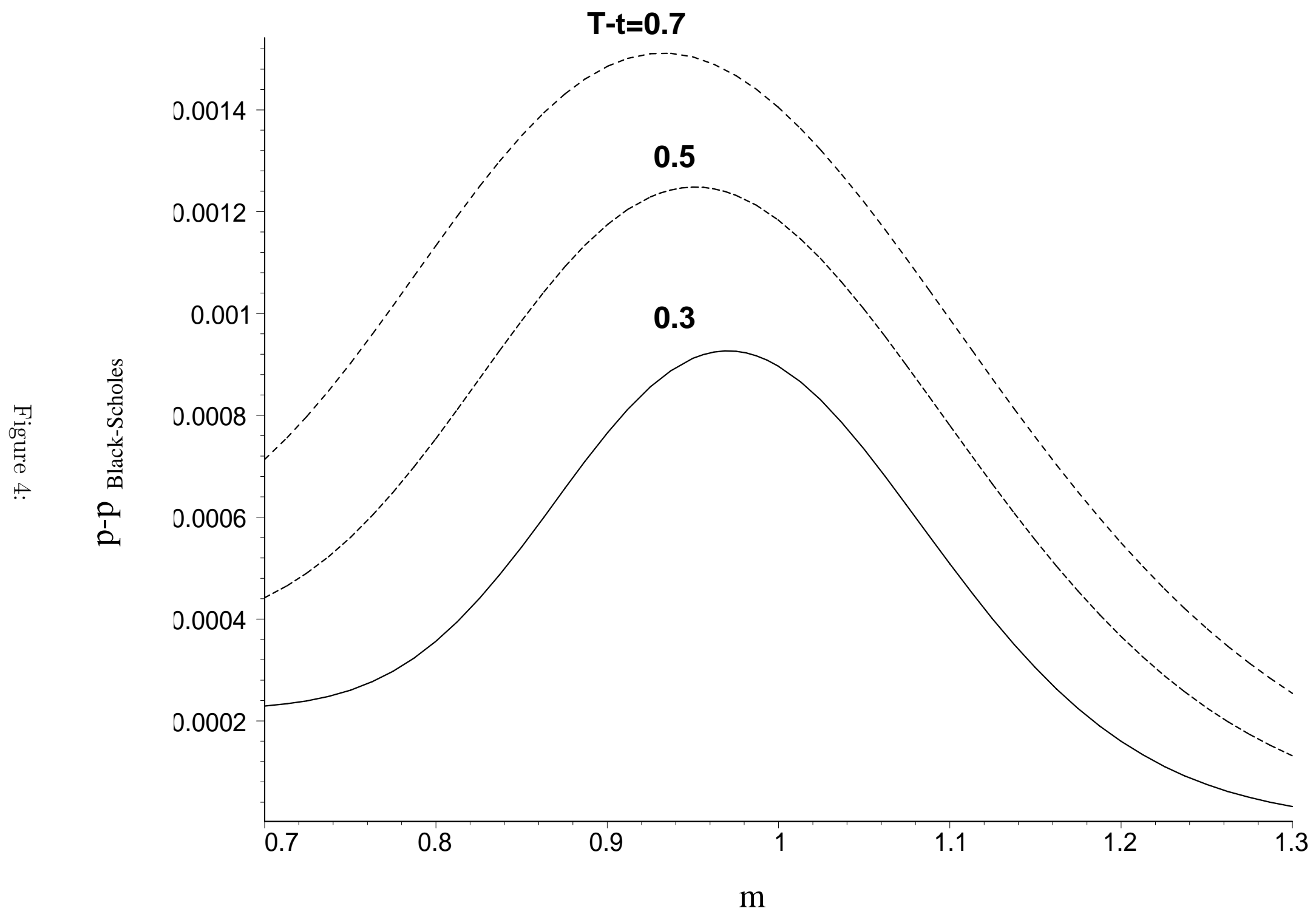

